\title{
APLICAÇÃO DO MÉTODO AHP TOPSIS 2N COMO APOIO À TOMADA DE DECISÃO: ESTUDO DE CASO NO DEPARTAMENTO JURÍDICO DA SECRETARIA MUNICIPAL DE SAÚDE DE DUQUE DE CAXIAS
}

\author{
Nathália Campos Oliveira (UNIGRANRIO) nathaliacampos3@unigranrio.br \\ Pamela Ferreira Carvalho de Oliveira (UNIGRANRIO) pamelaolivera@unigranrio.br \\ Carlos Francisco Simões Gomes (UFF) cfsg1@ @ol.com.br \\ Prof. Dr. Marcos dos Santos (Instituto Militar de Engenharia - IME) \\ marcosdossantos_doutorado_uff@yahoo.com.br
}

\section{Resumo}

A evolução recente das tecnologias digitais trouxe grandes possibilidades de melhoria para a humanidade. Pode-se dizer que um dos equipamentos advindos dessa evolução tecnológica é a impressora, instrumento capaz de auxiliar os usuários em suas tarefas relacionadas ao ambiente profissional ou cotidiano. O objetivo deste estudo é de ranquear um conjunto de alternativas de impressoras disponíveis no mercado que atendam aos critérios préestabelecidos pelos colaboradores do setor jurídico da Secretaria de Saúde de Duque de Caxias. A metodologia utilizada foi o AHP TOPSIS $2 \mathrm{~N}$ que é um método híbrido constituído por duas técnicas de tomada de decisão multicritério que são usualmente adotadas em cenários complexos, caracterizados pela presença de objetivos múltiplos e conflitantes. Ao aplicar a metodologia foi possível selecionar o modelo de impressora que mais se aproximou dos requisitos elencados pelo decisor sendo a impressora da marca HP LaserJet M402DN a mais adequada para a aquisição. Dessa forma, é possível dizer que essa ferramenta possui uma gama de aplicações, sendo capaz de contribuir no processo de tomada de decisão em diferentes seguimentos.

Palavras-Chaves: Tomada de Decisão; Multicritério; Método AHP TOPSIS 2N; Impressoras; Departamento Jurídico.

\section{Introdução}

A evolução recente das tecnologias digitais trouxe grandes possibilidades de melhoria para a humanidade. Pode-se dizer que um dos equipamentos advindos dessa evolução tecnológica é a impressora, instrumento capaz de auxiliar os usuários em suas tarefas relacionadas ao ambiente profissional ou cotidiano.

Seguindo a ideia da máquina de escrever, a primeira impressora foi projetada em 1938 por Chester Carlson, um americano que desenvolveu o processo de reprodução de imagens e textos por meio de fotocopiadoras. 
Em 1953 foi criada a primeira impressora de alta velocidade para a época. A mesma foi utilizada no primeiro computador comercial fabricado, desenvolvido nos Estados Unidos, o UNIVAC. As primeiras impressoras processavam a impressão através de impacto, o que acabava produzindo documentos de baixa qualidade, como trabalhava as impressoras matriciais.

As impressoras foram sendo aperfeiçoadas na mesma proporção que os computadores, e com o tempo foram sendo desenvolvidas impressoras para fins específicos, assim como os computadores.

Em 1983 surgiram as impressoras a laser, criadas pelo trabalho em conjunto das empresas Hewllet Packard e Canon. Esse dispositivo utilizava um laser especial para impressão, o que melhorou muito a qualidade da impressão em relação à tinta, que nas outras impressoras ficavam molhadas e acabavam escorrendo, o que não acontecia nesses novos modelos. As imagens e documentos impressos por esses dispositivos são de qualidade boa à excelente.

As impressoras a jato surgiram na década de 80 e, segundo Morimoto (2005), estas se tornaram uma tecnologia dominante na área de impressão de cores, pois são mais rápidas, possuem uma boa qualidade e resolução comparada à impressora a laser. A impressora a jato funciona lançando minúsculas gotas de tinta sobre o papel para criar uma imagem, com pontos muito pequenos, em média de 10 a 30 pontos por milímetro. Pode- se notar que os pontos são colocados com muita precisão, o que deixa a impressão com ótima qualidade.

De acordo com Camargo Júnior et al. (2010), as empresas estão gradativamente utilizando recursos computacionais em busca de flexibilidade, produtividade e redução de custos. Diante disso, fica evidente a real necessidade de se compreender como novas tecnologias estão mudando a rotina das empresas e auxiliando-as na obtenção de resultados satisfatórios mediante a incorporação das novas tecnologias.

\section{Descrição do problema}

A Secretaria Municipal de Saúde da cidade de Duque de Caxias localizada no estado do Rio de Janeiro possui como atribuição desenvolver, orientar, coordenar e executar a política de saúde do município, averiguando tanto a prevenção quanto o cuidado ambulatorial e hospitalar, bem como a legislação vigente.

Dentre os diversos departamentos existentes na Secretaria existe o departamento jurídico, o mesmo é responsável pelas questões legais que envolvem a população e a própria Secretaria. 
O setor recentemente está passando por algumas dificuldades em relação ao atual equipamento de impressão no qual em certos momentos de utilização ocorre o atolamento de papel e consequentemente paralisa o funcionamento da impressora, em outras situações a fila de impressão fica em estado de pausa causando muitos transtornos na rotina de trabalho. Além disso, há frequentemente vazamentos de tinta ou manchas indevidas de impressão. Com isso, esses eventos acabam muitas das vezes atrapalhando o desenvolvimento do trabalho dos funcionários deste setor posto que os mesmos acabam perdendo muito tempo tentando resolver o problema ou quando não conseguem retomar o funcionamento do equipamento o setor deve aguardar a visita do técnico responsável.

É evidente a importância das impressoras nos dias atuais. Seus mecanismos proporcionam a impressão de documentos com uma imensa rapidez e facilidade, ideia que vem desde as arcaicas máquinas de escrever. Nesse sentido, é fácil perceber que, de uma forma ou de outra, a impressora foi uma espécie de resultado da modernização do antigo instrumento.

Os dispositivos de impressão evoluíram conforme o avanço da própria computação. Da mesma forma que foram surgindo computadores destinados a diferentes usos, também foram criadas impressoras destinadas a fins específicos.

Existem diferentes tipos de impressoras no mercado e cada modelo possui suas funções específicas para atenderem suas demandas. Alguns tipos de impressoras utilizadas atualmente são as de jato de tinta, modelo mais antigo e comum que utiliza cartuchos de tinta para impressão possuindo qualidade de impressão variando de baixa para média impressão. Além deste modelo tem-se as impressoras à laser que utilizam laser para imprimir e possuem qualidade de imagem variando de boa à excelente dependendo da marca. Ademais há no mercado a impressora 3D que possui muita utilidade para moldes de produtos. A mesma cria objetos em 3D a partir de um programa no computador, que faz o design do objeto.

Com o advento das novas tecnologias as impressoras passaram a desempenhar diferentes funções que vão muito além da função de imprimir papéis. Dessa forma, são utilizadas impressoras que combinem a impressão tradicional com funcionalidades essenciais para o mundo digital, como edição instantânea e arquivos, digitalizações, impressão em nuvem além de envio de documentos para qualquer dispositivo.

Paralelamente, o uso das impressoras é de suma importância para as diversas instituições que utilizam essa ferramenta no ambiente de trabalho, visto que precisam de documentos 
impressos para operações rotineiras, como comunicação com os clientes, manutenção de registros entre outros.

Para o presente trabalho conforme apresentando anteriormente o departamento jurídico da Secretaria de Saúde vem tendo dificuldades para realizar as impressões diárias. Por se tratar de um órgão público verifica-se que para a problemática em questão não seria viável realizar a terceirização das impressoras, mesmo que pudesse trazer melhores benefícios. Nesse sentido, o departamento optou por realizar a licitação de um equipamento novo que atenda as suas necessidades operacionais.

De acordo com o mapa mental apresentado na Figura 1 verificam-se as condições de contorno do problema. O mesmo tem por objetivo organizar e analisar o conteúdo de forma mais compreensível.

Figura 1: Mapa mental com as condições de contorno do problema

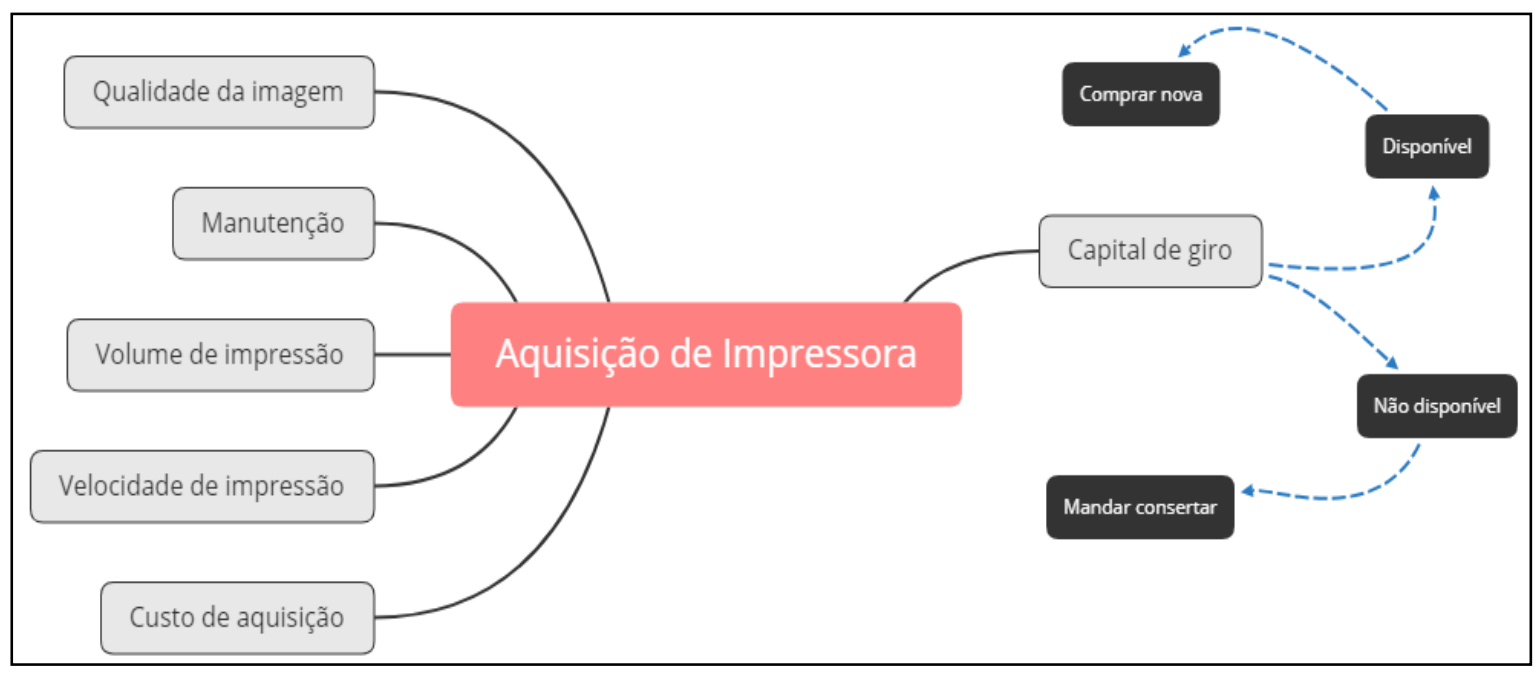

Fonte: Autores (2019)

Dado o exposto, esta pesquisa visa responder a seguinte questão: como selecionar uma impressora multifuncional que atenda às necessidades do setor jurídico da Secretaria Municipal de Saúde da cidade de Duque de Caxias?

\section{Fundamentação teórica}

\subsection{Pesquisa operacional}

A Pesquisa Operacional (PO) auxilia na resolução de problemas e na otimização de processos por meio de modelos matemáticos, desempenhando assim um planejamento estratégico competitivo.

O início da atividade, assim denominada Pesquisa Operacional, geralmente é atribuída às atividades militares nos primórdios da Segunda Guerra Mundial. Isso porque, surgiu como 
resultado de estudos de equipes interdisciplinares de cientistas contratados para resolver os problemas militares de ordem tática e estratégica.

Nas fases para o estudo da Pesquisa Operacional primeiramente é definido o problema e feito a coleta de dados, para então formular um modelo matemático o qual representará o problema a ser analisado (HILLIER; LIEBERMAN, 2013).

A aplicação da Pesquisa Operacional traz diversos resultados positivos na administração de empresas possuindo uma enorme variedade de aplicações do seu modelo. A mesma possui diferentes funções podendo ser aplicada em diversas áreas como transportes, manufatura, planejamento financeiro, serviços públicos, entre outros.

\subsection{Métodos multicritério para tomada de decisão}

Os métodos multicritério de tomada de decisão surgiram como métodos de apoio que são compreendidos como ferramentas matemáticas, eficazes para solução de problemas em que existem critérios conflitantes (BRANS \& MARESCHAL, 2005).

A abordagem multicritério de apoio à decisão caracteriza-se como um grupo de métodos que buscam evidenciar um problema, no qual as alternativas são avaliadas por múltiplos critérios, sendo na maioria dos casos de caráter divergente (GOMES et al, 2002).

Os métodos multicritério para apoio à decisão possuem aspectos científicos e, ao mesmo tempo, subjetivo, com capacidade de agregar todas as características consideradas importantes, inclusive as não quantitativas, com o intuito de possibilitar a transparência e sistematização do processo pertinente aos problemas de tomada de decisões.

De modo geral, problemas complexos de tomada de decisão, de acordo com (GOMES; GOMES, 2002) apresentam pelo menos uma das características a seguir:

- Os critérios para resolução do problema são divergentes entre si.

- Tanto os critérios quanto as alternativas não estão definidas de forma objetiva, e as consequências da escolha de uma alternativa em relação à pelo menos um critério, não são devidamente compreendidas.

- Os critérios e as alternativas podem estar interligados, de forma que um critério pode refletir nos demais.

- A solução dos problemas depende de um conjunto de pessoas, cada uma com pontos de vistas próprios. 
- As restrições dos problemas não estão bem definidas, podendo existir dúvidas em relação ao que é critério e ao que é restrição.

- Existem critérios quantificáveis, e outros que se tornam quantificáveis apenas através de juízos de valor efetuados sobre uma escala.

- A escala para um critério pode ser cardinal, verbal ou ordinal, dependendo da disponibilidade de dados e da natureza dos critérios.

\subsection{Método Analytic Hierarchy Process (AHP)}

O método AHP foi um dos primeiros métodos de tomada de decisão com múltiplos critérios. Este surgiu durante a década de 1970 e foi desenvolvido pelo matemático Thomas L. Saaty.

O método tem como finalidade organizar os objetivos ou critério numa hierarquia representada pela preferência dos decisores. Além de o método AHP possibilitar a resolução de problemas com critérios conflitantes, uma das vantagens apontadas por Gomes (2009) se deve ao fato de o método permitir a participação de múltiplas pessoas, visto que é possível envolver diversos critérios e alternativas.

A utilização do método AHP é também indicada pelo fato de mensurar critérios tangíveis com intangíveis, através de uma escala de razão, podendo o problema ser divido em diversas partes, relacionando-as e conectando os juízos de comparação com o objetivo final da aplicação (VARGAS, 1990).

Saaty (1980) propôs o uso de uma escala padrão de valores, variando de 1 a 9 , como forma de avaliar numericamente alternativas e critérios em um processo de decisão. Conforme Figura 2 para cada valor, Saaty preocupou-se em definir seu predicado qualitativo seguido de explicação textual, para diminuir dúvidas no momento do julgamento.

Figura 2: Escala fundamental de Saaty

\begin{tabular}{|c|l|l|}
\hline Valor & \multicolumn{1}{|c|}{ Definição } & \multicolumn{1}{c|}{ Explicação } \\
\hline $\mathbf{1}$ & Igual impertância & $\begin{array}{l}\text { os dois critérios contribuem de forma idêntica } \\
\text { para o objetivo }\end{array}$ \\
\hline $\mathbf{3}$ & Pouco mais importante & $\begin{array}{l}\text { a análise e a experiência mostram que um critério } \\
\text { é um pouco mais importante que o outro }\end{array}$ \\
\hline $\mathbf{5}$ & Muito mais importante & $\begin{array}{l}\text { a análise e a experiência mostram que um critério } \\
\text { é claramente mais importante que o outro }\end{array}$ \\
\hline $\mathbf{7}$ & Bastante mais importante & $\begin{array}{l}\text { a análise e a experiência mostram que um dos } \\
\text { critérios é predominante para o objetivo }\end{array}$ \\
\hline $\mathbf{9}$ & $\begin{array}{l}\text { Extremamente mais } \\
\text { importante }\end{array}$ & $\begin{array}{l}\text { sem qualquer dúvida um dos critérios é } \\
\text { absolutamente predominante para o objetivo }\end{array}$ \\
\hline $\begin{array}{c}\mathbf{2 , 4 , 6 , 8} \\
\text { valores recíprocos } \\
\text { dos anteriores }\end{array}$ & Valores intermediários & também podem ser utilizados \\
\hline
\end{tabular}

Fonte: Saaty (1980) 


\subsection{Método Technique for Order Preferences by Similarity to Ideal Solution (TOPSIS)}

O TOPSIS foi proposto por Hwang e Yoon (1981) e parte do princípio que busca estabelecer uma relação em que uma determinada alternativa esteja mais próximo o possível de uma solução hipotética ideal e mais afastado o possível de uma solução oposta à ideal. Este método se destaca por ser intuitivo e pela simplicidade dos procedimentos matemáticos, facilidade de implementação e aplicação, e permite avaliar uma quantidade não limitada de alternativas (SOUZA et. al. 2016).

Nesse sentido, na Figura 3 é possível ilustrar um filtro que elimina algumas possibilidades de modelos diante de cada decisão tomada pelo gestor. Essas decisões estão ligadas à abordagem escolhida em uma etapa aleatória do processo ou em hipóteses assumidas em relação ao problema em estudo. Na passagem por cada filtro, há um número menor de possíveis formas de representar o problema, ou seja, os modelos, que são representados pelas esferas pretas. Alguns modelos podem nem ser percebidos pelo analista, que os elimina a partir das definições e hipóteses que vai formando ao longo do processo.

Figura 3: Filtro de modelos

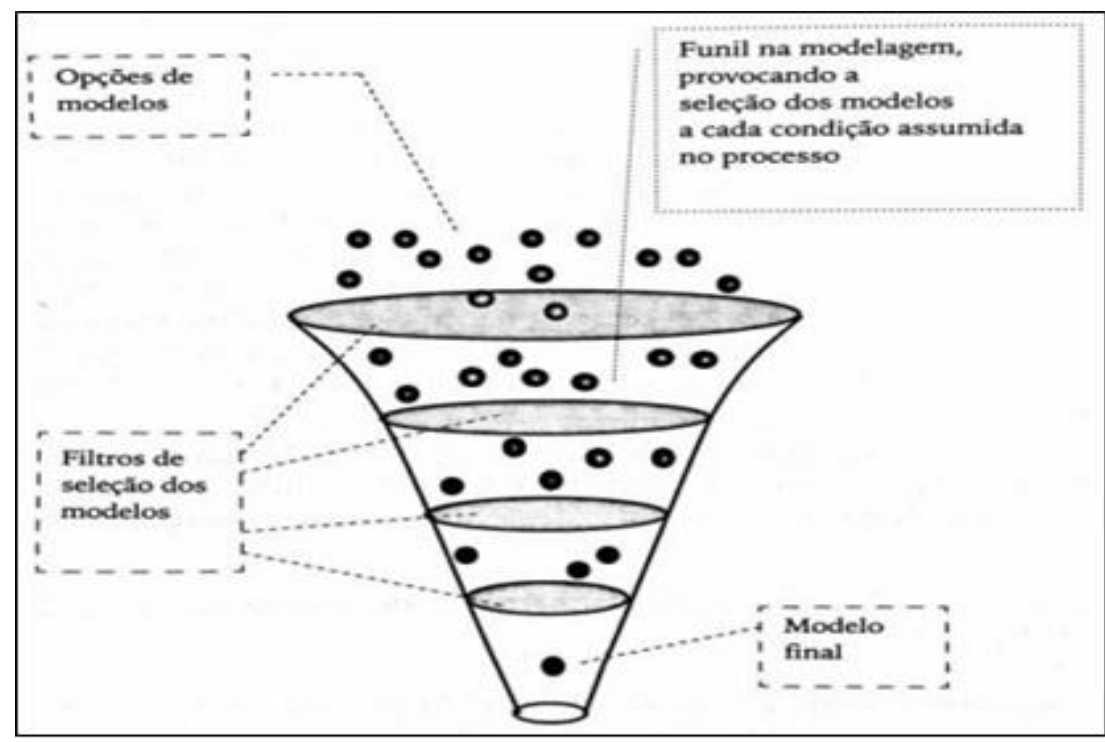

Fonte: Almeida (2013)

\subsection{Método AHP TOPSIS 2N}

O Método AHP TOPSIS $2 \mathrm{~N}$ é um método híbrido constituído por duas técnicas de tomada de decisão multicritério que são usualmente adotadas em cenários complexos, caracterizados pela presença de objetivos múltiplos e conflitantes. 
Alguns autores vêm utilizando métodos que une o AHP com o TOPSIS. Os resultados obtidos em (SOUZA et. al., 2018) mostraram que o método AHP TOPSIS 2N possui um largo potencial para lidar com problemas de priorização para seleção de equipamentos.

Para a aplicação do método em estudo são descritos os passos a seguir:

1. Definição da matriz de decisão: expressa a pontuação de cada alternativa em relação a cada critério, mostrando o quanto uma alternativa domina ou é dominada pelas demais;

2. Definição da matriz de ponderação: utilizando a Escala Fundamental de Saaty, através de grupo multidisciplinar cada critério é avaliado contra cada critério;

3. Determinação do peso de cada critério: através da aplicação do método AHP. É importante avaliar a razão de consistência e caso a mesma seja superior a 0,1 fazer uma nova avaliação dos critérios e uma nova matriz de ponderação;

4. Normalização da Matriz de Decisão: no caso do método AHP TOPSIS 2N são utilizadas duas diferentes normalizações (SOUZA et. al., 2018);

5. Construção da Matriz de Decisão Normalizada Ponderada: as matrizes ponderadas pelo $1^{\circ} \mathrm{e}$ $2^{\circ}$ normalização são ponderadas utilizando os pesos obtidos no passo 3 ;

6. Determinação da Solução Ideal Positiva (SIP) e da Solução Ideal Negativa (SIN);

7. Cálculo das medidas de distância: são obtidas medidas de distância euclidiana de cada uma das alternativas em relação a SIP e a SIN;

8. Cálculo da proximidade relativa à alternativa ideal: é obtida pela razão $\mathrm{R}$ igual a distância para o ponto SIN pela soma da distância ao ponto SIP e a distância para o ponto SIN;

9. Ordenação das preferências.

\section{Estudo de caso}

Para a realização do estudo de caso foi utilizado o método AHP TOPSIS $2 \mathrm{~N}$. No que tange ao levantamento dos critérios, foi realizada uma visita à Secretaria Municipal de Saúde a fim de coletar informações relevantes para o desenvolvimento do trabalho. Foi feita uma entrevista com os colaboradores do setor jurídico no qual foram elencados os critérios relevantes para a aplicação do método.

A partir das alternativas e dos critérios apresentados pelos colaboradores, foi possível realizar a construção da seguinte matriz de avaliação ilustrada na Tabela 1. 
Tabela 1: Matriz de avaliação

\begin{tabular}{lcccc}
\hline \multicolumn{1}{c}{ Impressoras } & $\begin{array}{c}\text { Custo de Aquisição } \\
\text { (R\$) }\end{array}$ & $\begin{array}{c}\text { Velocidade de } \\
\text { impressão } \\
\text { (Páginas Por } \\
\text { Minuto) }\end{array}$ & $\begin{array}{c}\text { Volume máximo } \\
\text { de Ciclo Mensal } \\
\text { (Quant. Páginas) }\end{array}$ & $\begin{array}{c}\text { Bandeja de papel } \\
\text { (Quant.) }\end{array}$ \\
\hline Brother DCP - L2540DW & 1.300 & 30 & 10.000 & 250 \\
HP LaserJet - M521 & 3.600 & 40 & 75.000 & 600 \\
Ricoh SP 377SFNWX & 1.500 & 30 & 35.000 & 300 \\
HP LaserJet - M402DN & 1.700 & 40 & 80.000 & 350 \\
\hline
\end{tabular}

Fonte: Autores (2019)

Assim foi possível listar 4 critérios considerados relevantes para o problema em questão:

- Custo de aquisição: este critério é de suma importância, pois envolve o valor em reais bruto para que seja possível efetuar a compra do equipamento.

- Velocidade de impressão: está relacionado a velocidade de impressão.

- Volume máximo: faz referência a quantidade de cópias por mês.

- Bandeja de papel: é a quantidade de folhas que é acomodada na impressora.

\subsection{Aplicação do método AHP TOPSIS 2N}

Os resultados do método AHP TOPSIS $2 \mathrm{~N}$ foram obtidos a partir de um software desenvolvido em Python por Ribeiro, Santos e Gomes (2019), no Instituto Militar de Engenharia.

O início da aplicação do método é realizado a partir da inserção dos critérios relevantes, em seguida é atribuído os valores relacionados aos critérios no qual são baseados a partir da Escala de Saaty e por fim é gerada a matriz de ponderação. A Figura 4 demostra esta etapa de aplicação.

Figura 4: Matriz de ponderação

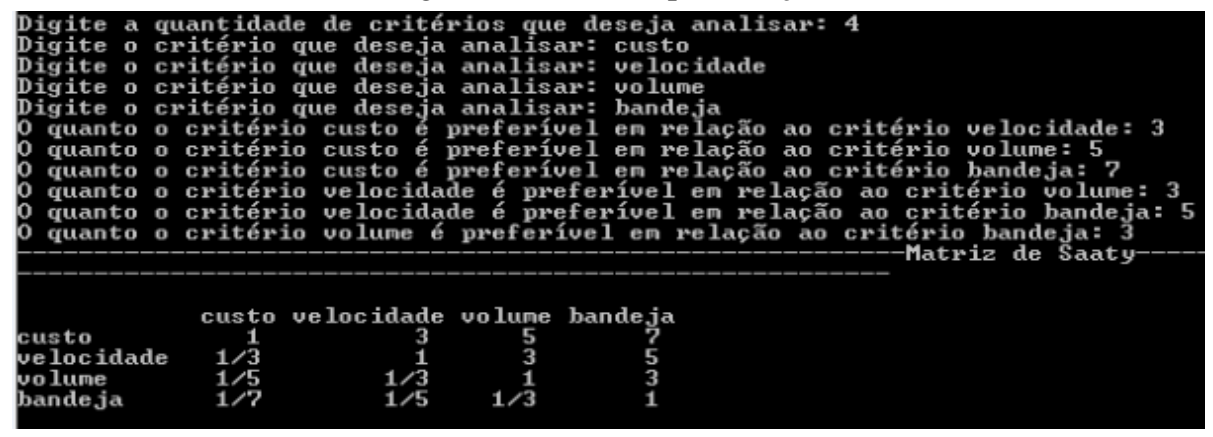

Fonte: Autores (2019) 
Após a elaboração da matriz de ponderação são determinados os pesos de cada critério conforme Figura 5.

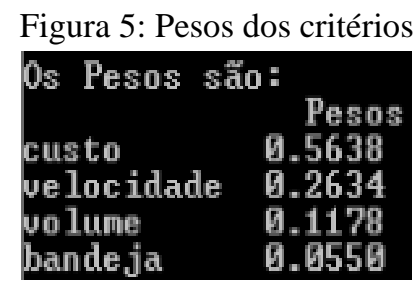

Fonte: Autores (2019)

Como indicador de coerência entre as matrizes, foi calculado o Lambda máx, a Razão de Consistência (RC) e o Índice de Consistência (IC). A partir dessas informações foi possível perceber conforme Figura 6 que o valor de RC foi menor do que 10\%, não sendo necessário rever os dados de comparações, ou seja, os dados estão consistentes para continuidade da aplicação do método.

Figura 6: Índice de coerência

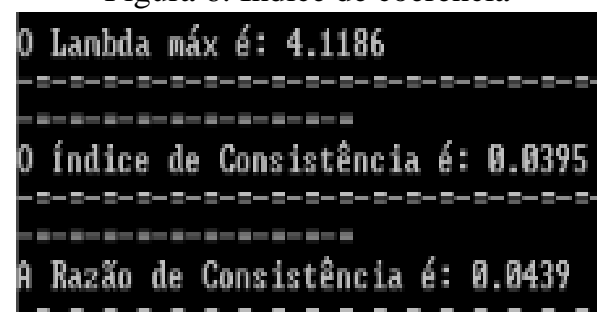

Fonte: Autores (2019)

Conforme Figura 7 foram atribuídos os dados relacionados as alternativas e aos critérios.

Figura 7: Alternativas e critérios da matriz de decisão

\begin{tabular}{|c|c|c|c|c|c|}
\hline $\begin{array}{l}\text { Brother DCP } \\
\text { HP Laserjet } \\
\text { Ricoh SP } \\
\text { HP Laserjet }\end{array}$ & $\begin{array}{l}\text { M521 } \\
\text { M462DN }\end{array}$ & $\begin{array}{r}\text { custo } \\
1300.0 \\
36000.0 \\
1500.0 \\
1700.0\end{array}$ & $\begin{array}{r}\text { ve locidade } \\
30.0 \\
40.0 \\
30.0 \\
40.0\end{array}$ & $\begin{array}{r}\text { volume } \\
10006.0 \\
75000.0 \\
35000.0 \\
80000.0\end{array}$ & $\begin{array}{r}\text { bande.ja } \\
250.6 \\
600.0 \\
300.0 \\
350.6\end{array}$ \\
\hline
\end{tabular}

Fonte: Autores (2019)

Posteriormente foi gerada a primeira normalização de acordo com a Figura 8.

Figura 8: Primeira normalização

\begin{tabular}{lrrrr} 
& custo & velocidade & volume & bandeja \\
Brother DCP & 0.0366 & 0.4243 & 0.0865 & 0.3137 \\
HP Laser.jet H521 & 0.9974 & 0.5657 & 0.6491 & 0.7529 \\
Ricoh 8P & 0.0416 & 0.4243 & 0.3029 & 0.3765 \\
HP Laserjet M462DN & 0.0471 & 0.5657 & 0.6924 & 0.4392 \\
\hline
\end{tabular}

Fonte: Autores (2019) 
Em seguida a segunda normalização segundo a Figura 9.

Figura 9: Segunda normalização

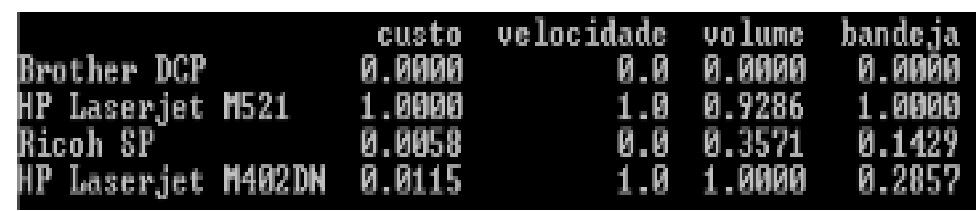

Fonte: Autores (2019)

Na Figura 10 é ilustrada a primeira normalização ponderada.

Figura 10: Primeira normalização ponderada

\begin{tabular}{lrrrr} 
& custo & velocidade & volume & bandeja \\
Brother DCP & 0.0203 & 0.1118 & 0.0102 & 0.0173 \\
IP Laserjet H521 & 0.5623 & 0.1490 & 0.0765 & 0.0414 \\
Ricoh SP & 0.0235 & 0.1118 & 0.0357 & 0.02 .07 \\
HP Laserjet H462DN & 0.0266 & 0.1490 & 0.0816 & 0.0242 \\
\hline
\end{tabular}

Fonte: Autores (2019)

Na Figura 11 é demostrada a segunda normalização ponderada.

Figura 11: Segunda normalização ponderada

\begin{tabular}{|c|c|c|c|c|}
\hline $\begin{array}{l}\text { DCP } \\
\text { rjet } \mathrm{M} 521 \\
\mathrm{P} \\
\text { rjet } \mathrm{M} 402 \mathrm{DN}\end{array}$ & $\begin{array}{l}\text { custo } \\
0.0906 \\
0.5638 \\
0.0933 \\
0.0365\end{array}$ & $\begin{array}{r}\text { ve locidade } \\
0.6090 \\
8.2634 \\
8.6990 \\
6.2634\end{array}$ & $\begin{array}{l}\text { volume } \\
0.0809 \\
0.1094 \\
0.0421 \\
0.1178\end{array}$ & $\begin{array}{r}\text { bande ja } \\
0.0009 \\
0.0550 \\
0.0579 \\
0.0157\end{array}$ \\
\hline
\end{tabular}

Fonte: Autores (2019)

Segundo a Figura 12 é feita a determinação da solução ideal positiva e solução ideal negativa no qual se tem como max para valor máximo e min para valor mínimo.

Figura 12: Determinação da SIP e SIN

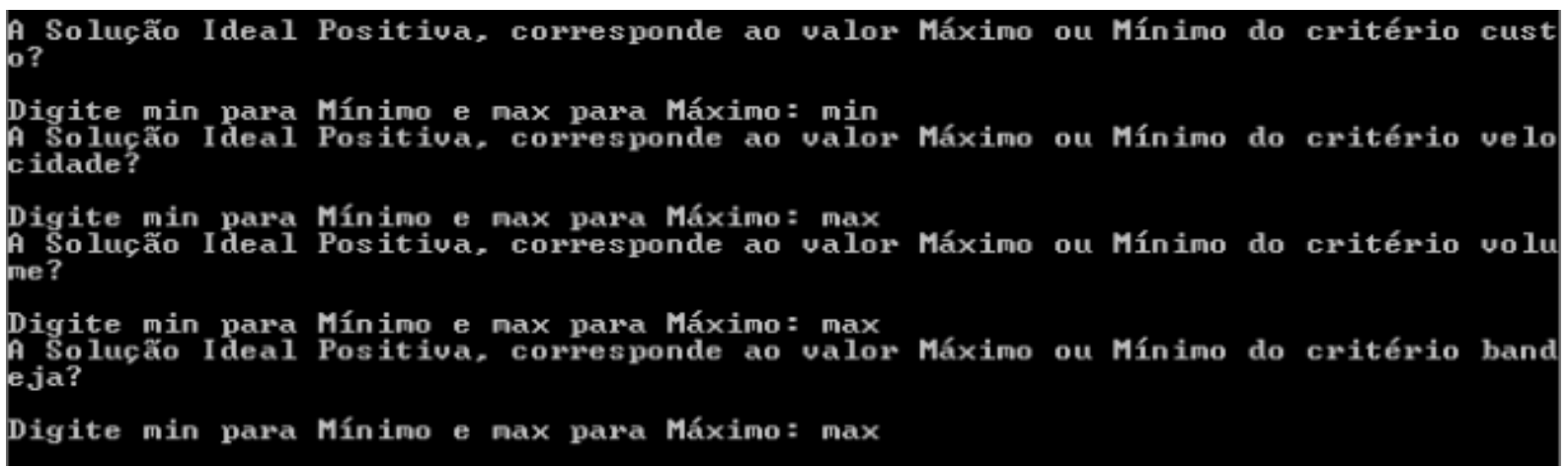

Fonte: Autores (2019)

\section{Resultados alcançados}

Após a aplicação do método AHP TOPSIS 2N, é observado na Figura 13 e na Figura 14 respectivamente os resultados de priorização referente à primeira e à segunda normalização. É 
possível notar que a impressora HP Laserjet M402DN foi escolhida como a melhor opção dentre os outros modelos. Vale ressaltar que este modelo ficou na primeira classificação em ambos resultados podendo então dizer que o equipamento possivelmente irá ser adquirido e em consequência disso auxiliando na solução do problema em estudo.

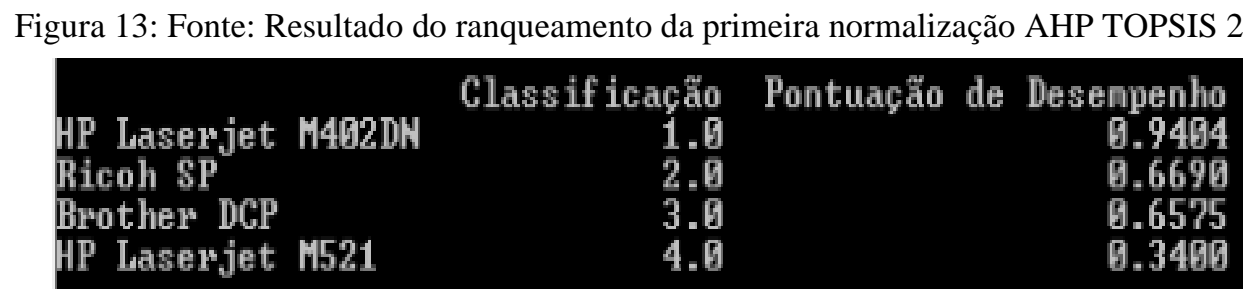

Fonte: Autores (2019)

Figura 14: Resultado do ranqueamento da segunda normalização AHP TOPSIS 2N

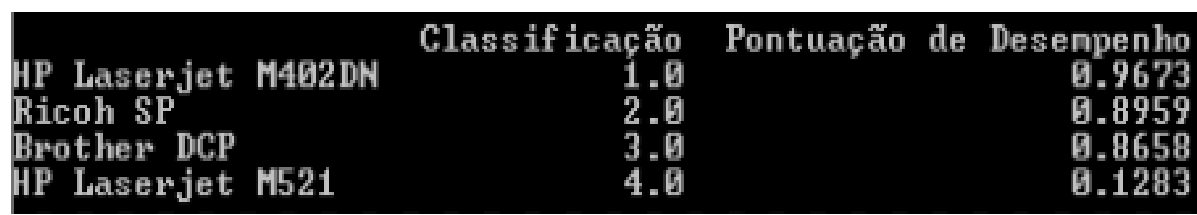

Fonte: Autores (2019)

\section{Considerações finais}

Conforme foi observado no presente trabalho, o método AHP TOPSIS $2 \mathrm{~N}$, aplicado em diferentes situações, mostrou-se adequado na modelagem do problema abordado no estudo de caso.

Primeiramente, foi identificado o problema e constatou-se a necessidade de adquirir uma nova impressora que fosse capaz de funcionar corretamente no setor jurídico da Secretaria de Saúde de Duque de Caxias. Por meio do método, foi possível obter a classificação final da impressora que teve melhor resultado dentre os quatros modelos apresentados no presente estudo.

Através do resultado esperado, o objetivo desta ferramenta é auxiliar na tomada de decisão de uma forma clara e objetiva buscando assim contribuir na resolução de um determinado problema. Levando em consideração a ferramenta estudada é possível notar sua gama de aplicabilidade. Pode ser utilizada para resolução de problemas dos mais variados tipos, sendo assim um método de grande utilidade voltado para a contribuição da tomada de decisão.

Em virtude dos fatos mencionados, com a aplicação do método AHP TOPSIS 2N, conseguiuse propor uma metodologia de auxílio na tomada de decisão, para que os colaboradores tenham um maior embasamento analítico, técnico e quantitativo na seleção da impressora a ser adquirida. 


\section{Referências}

Brans, J. P., \& Mareschal, B. (2005). Multiple criteria decision analysis - state of the art. Dordrecht: Kluwer Academic Publishers.

CAMARGO JÚNIOR, J. B de.; PIRES, S. R. I; SOUZA, A. H. R. SISTEMAS INTEGRADOS DE GESTÃO ERP E CLOUD COMPUTING: características, vantagens e desafios.In: SIMPÓSIO DE ADMINISTRAÇÃO DA PRODUÇÃO, LOGÍSTICA E OPERAÇÕES INTERNACIONAIS 2010. 2010, São Paulo. Anais...São Paulo: SIMPOI, 2010. 13 p. CD-ROM.

COLOMBO, Danilo; SANTOS, Marcos dos; GOMES, Carlos Francisco Simões. Seleção da melhor configuração de poço de petróleo para o desenvolvimento de um campo: utilizando uma ferramenta de apoio multicritério. In: SIMPÓSIO DE PESQUISA OPERACIONAL E LOGÍSTICA DA MARINHA, 19., 2019, Rio de Janeiro, RJ. Anais [...]. Rio de Janeiro: Centro de Análises de Sistemas Navais, 2019.

Gomes, L. F. A. M.; Gomes, C. F. S. \& Almeida, A. T. (2002). Tomada de Decisão Gerencial: Enfoque Multicritério. Editora Atlas, Rio de Janeiro.

HILLIER, F. S.; LIEBERMAN G. J. Introdução à Pesquisa Operacional. 9. ed. Porto Alegre: AMGH, 2013.

MORIMOTO, Carlos E., Impressora Jato de Tinta. 26 de jun. 2005. Disponível em: <http://www.hardware.com.br/termos/impressora-jato-de-tinta>. Acesso em: 19 nov. 2019.

SAATY, Thomas L. The Analytic Hierarchy Process. New York: McGraw-Hill International, 1980.

Souza, L. P., Barros, A. P., Gomes, C. F. S. (2016) Comitê de Governança de TI: um modelo Decisório para Ordenamento e Priorização de Portfólio de Projetos de Investimento por Método Híbrido de Técnicas de Auxílio Multicritério, XIII SEGeT, Simpósio de Excelência em Gestão e Tecnologia.

Souza, L.; Gomes, C. F. S.; Barros, A. (2018). Implementation of new Hybrid AHPTOPSIS$2 \mathrm{~N}$ method in sorting and prioritizing of an it capex project portfolio. International Journal of Information Technology \& Decision Making. 10.1142/S0219622018500207.

RIBEIRO, Wallace Lima; SANTOS, Marcos dos; GOMES, Carlos Francisco Simões. AHPTOPSIS-2N Software (v.1). Desenvolvido por meio de uma parceria entre o Institulo Militar de Engenharia (IME), o Centro de Análises de Sistemas Navais (CASNAV) e a Universidade Federal Fluminense (UFF), 2019.

Vargas, L. G. (1990). An overview of the Analytic Hierarchy Process and its applications. European Journal of Operational Research, 48(1), 2-8. http:// dx.doi.org/10.1016/03772217(90)90056-H. 